\title{
Clasificación cuantitativa de la maniobra de pivot-shift
}

\author{
Quantitative classification of pivot-shift maneuver \\ Berumen-Nafarrate E, ${ }^{*}$ Carmona-González J, ${ }^{\ddagger}$ Tonche-Ramos JJ, ${ }^{\ddagger}$ \\ Carmona-Máynez O,* Aguirre-Madrid A, * Reyes-Conn RA, * Ramos-Moctezuma IR, ${ }^{\ddagger}$ \\ Sigala-González LR, ${ }^{\ddagger}$ Quintana-Trejo FN,${ }^{\ddagger}$ Moreno-Brito V,${ }^{\ddagger}$ Leal-Berumen I ${ }^{\ddagger}$ \\ Hospital Christus Mugerza del Parque. Chihuahua, Chihuahua.
}

\begin{abstract}
RESUMEN. Introducción: El diagnóstico de lesión del ligamento cruzado anterior se establece con la exploración física que incluya la maniobra de Lachman y la prueba de pivot-shift, ya que cuenta con el mayor valor predictivo positivo. Aún se recurre a pruebas de gabinete como la RMN para la confirmación del mismo; por lo que se busca la cuantificación de maniobras clínicas. Material y métodos: Estudio clínico prospectivo en el que se cuantifican las maniobras de pivot-Shift y Lachman para su posterior clasificación. Resultados: De 36 pacientes, 64\% se encontró un rango entre 0 y $3 \mathrm{~mm}$ al restar al valor de la rodilla sana el valor de la rodilla afectada. Se encontró que $28 \%$ de los pacientes, registraron valores de KT-1000, que oscilan entre los 4 y 6 mm de desplazamiento. En $8 \%$ del total de los pacientes se encontraron valores de KT-1000 que superaron los $7 \mathrm{~mm}$ de desplazamiento. Se normalizan los datos obtenidos y se clasifican en 3 grados de lesión: grado 1 con lesión parcial; grado 2 con lesión completa de haz anteromedial y parcial en haz posterolateral; grado 3 con lesión completa de ambos haces. Conclusiones: Se propone una clasificación para los grados de lesión del LCA con futura aplicación a una reconstrucción más personalizada.
\end{abstract}

Palabras clave: Ligamento cruzado, medición, inestabilidad, diagnóstico, clasificación.
ABSTRACT. Introduction: The diagnosis of ACL injury is established with the physical examination that includes the Lachman maneuver and the pivot-shift test since it has the highest positive predictive value. Imaging tests such as MRI are still used for confirmation of the same; so the quantification of clinical maneuvers is sought. Material and methods: Prospective clinical study in which the maneuvers of pivot-shift and Lachman are quantified for later classification. Results: Of 36 patients, 64\% found a range between 0 and $3 \mathrm{~mm}$ when subtracting the value of the affected knee from the value of the healthy knee. It was found that $28 \%$ of the patients recorded values of KT-1000 ranging between 4 and $6 \mathrm{~mm}$ of displacement. In $8 \%$ of the total patients, values of KT-1000 were found that exceeded $7 \mathrm{~mm}$ of displacement. The data obtained are normalized and classified into 3 degrees of injury: grade 1 with partial injury; grade 2 with complete anteromedial and partial injury in posterolateral bundle; grade 3 with complete injury of both bundles. Conclusion: A classification is proposed for the degrees of ACL injury with future application to a more personalized reconstruction.

Keywords: Cruciate ligament, measurement, instability, diagnosis, classification.

\section{Nivel de evidencia: IV}

* Ortopedia y Traumatología, Hospital Christus Mugerza del Parque. Chihuahua, Chihuahua.

‡ Facultad de Medicina y Ciencias Biomédicas, Universidad Autónoma de Chihuahua.

Correspondencia:

Irene Leal-Berumen

Facultad de Medicina y Ciencias Biomédicas, Universidad Autónoma de Chihuahua, Chihuahua, México.

Tel. (614) 4103320

E-mail: ileal@uach.mx

Recibido: 31-06-2019. Aceptado: 12-12-2020.

Citar como: Berumen-Nafarrate E, Carmona-González J, Tonche-Ramos JJ, Carmona-Máynez O, Aguirre-Madrid A, Reyes-Conn RA, et al. Clasificación cuantitativa de la maniobra de pivot-shift. Acta Ortop Mex. 2021; 35(2): 153-157. https://dx.doi.org/10.35366/101858 


\section{Introducción}

El diagnóstico de lesión del ligamento cruzado anterior (LCA) se establece con la exploración física que incluya la maniobra de Lachman y la prueba de pivot-shift, ya que cuenta con el mayor valor predictivo positivo. Una historia clínica detallada para conocer el mecanismo de lesión y la exploración física nos generará diagnósticos adicionales si existen como lesiones meniscales, inestabilidad del compartimento interno y de los ligamentos cruzados. Desde 1976 se ha hecho énfasis en la relación de ruptura de LCA y compromiso del compartimento interno ${ }^{1,2,3}$ y se complementa con auxiliares diagnósticos como radiografías simples de rodilla que, por sí solas, no integran el diagnóstico, sino que otorgan datos indirectos como lesiones por avulsión. La resonancia magnética es el estudio de gabinete de elección para corroborar una lesión de LCA, visualizando mejor la lesión en la secuencia T2 y densidad de protones con supresión grasa, además de ser útil para identificar lesiones asociadas como roturas meniscales o lesiones condrales. El grado de sensibilidad de la misma, para detectar lesión del LCA es de $86.5 \%$ y tiene una especificidad de $95.2 \%$. $3,4,5,6,7,8,9,10$

Existen algunos cuestionarios que permiten valorar de forma subjetiva la estabilidad de la rodilla, entre los que se incluyen:

Escala Tegner Lysholm: clasifica el grado subjetivo de los pacientes en relación con la capacidad funcional. ${ }^{11}$

Cuestionario IKDC: consta de una parte subjetiva que consiste en 18 preguntas que toman en cuenta síntomas, actividades deportivas y función, de las cuales al menos 16 deben ser respondidas para poder ser utilizado. ${ }^{12}$

Existen maniobras cuantitativas para valorar la integridad del LCA, mediante el uso de instrumentos como el KT-1000 que cuantifica la maniobra de Lachman y el pivotshift meter que pretende cuantificar la maniobra de pivotshift. ${ }^{13,14,15,16,17,18,19,20}$

Algunos otros dispositivos han sido utilizados para la medición del pivot-shift como sistemas electromagnéticos de rastreo, acelerómetros, sistemas de navegación y estudios imagenológicos. ${ }^{16}$ Los sistemas electromagnéticos de rastreo has diferenciado cambios mínimos en la cuestión rotacional y detectado el pivot-shift; sin embargo, hay algunas condiciones que pueden alterar el resultado como: estructuras metálicas dentro de la sala de exploración, dispositivos

Tabla 1: Clasificación de inestabilidad anterior de rodilla con uso de KT-1000.

Grados de acuerdo al KT-1000

$\begin{array}{ll}1 & 0-3 \\ 2 & 3-6 \\ 3 & >7\end{array}$

\begin{tabular}{|c|c|c|c|}
\hline & $\begin{array}{l}\text { Rangos } \\
\text { o de acu }\end{array}$ & $\begin{array}{l}\text { os en cadt } \\
\text { is grados }\end{array}$ & \\
\hline \multirow[b]{2}{*}{ Grado } & \multicolumn{3}{|c|}{ Acelerómetros } \\
\hline & Eje $X$ & Eje Y & Eje Z \\
\hline 1 & 75-90* & $60-94 *$ & $60-74 *$ \\
\hline 2 & 91-95* & $95-100 *$ & $75-85^{*}$ \\
\hline 3 & $\geq 96^{*}$ & $\geq 100^{*}$ & $\geq 86^{*}$ \\
\hline
\end{tabular}

electrónicos, la movilidad de tejidos blandos al realizar la maniobra. ${ }^{16,21}$ Los sistemas de navegación son precisos en los resultados obtenidos de traslación y rotación, pero son costosos y poco disponibles en los centros de salud. ${ }^{16}$

KT-1000 Medmetric Knee Ligament Arthrometer. Es el instrumento considerado «estándar de oro» en la medición del desplazamiento de la rodilla con insuficiencia del LCA. Mediante la utilización de un artrómetro se evalúa el desplazamiento anterior de la tibia sobre el fémur midiendo la maniobra de «cajón anterior» en milímetros. Si el resultado fuera mayor a $11 \mathrm{~mm}$ en una sola pierna o una diferencia mayor de $3 \mathrm{~mm}$ entre ambas rodillas se considera positivo para la lesión del LCA; no obstante, algunos estudios reportan hasta $28 \%$ de falsos negativos con el uso de KT$1000 / 2000 .^{6,22,23}$

Ademas del KT-1000 los dispositivos de laxitud más utilizados son Telos, GNRB $^{\circledR}$, Rolimeter $^{\circledR}$, en los cuales se han hecho estudios para comparar los resultados entre si y hay poca correlación entre ellos, lo que concluye que pueden ser utilizados en conjunto pero no comparándolos. 24,25,26

Pivot-shift meter: dispositivo que consiste en un par de acelerómetros y un giroscopio unidos por bandas elásticas, las cuales se deben ajustar por encima de la rótula y sobre la tuberosidad tibial. Una vez ajustado el equipo se abre el programa en la computadora para registrar el movimiento de los acelerómetros durante la realización de la maniobra. ${ }^{6}$

Se han realizado algoritmos de tratamiento en lesiones de LCA y sus lesiones asociadas en tratamiento primario y en revisión de la misma reconstrucción tomando en cuenta lesiones meniscales, lesiones de esquina anterolateral y posterolateral y problemas de mal alineamiento coronal. Se busca actualmente realizar reconstrucciones anatómicas y añadir la reparación asociada de las lesiones agregadas del paciente; ${ }^{27}$ aunque hay estudios que reportan que los pacientes con hiperlaxitud no generan una relación al incremento rotacional de la rodilla afectada por lesión de LCA. ${ }^{28}$

En pacientes menores de 20 años se debe tener más precaución en el proceso de rehabilitación y de preferencia utilizar injertos para la reconstrucción entre 8 y 10 mm para prevenir el aflojamiento y posterior inestabilidad. ${ }^{29,30}$

Objetivo: desarrollo de una clasificación para el grado de lesión del LCA mediante la cuantificación de la maniobra del pivot-shift; tomando como base el estándar de oro KT-1000. 


\section{Material y métodos}

Estudio clínico, prospectivo de pacientes con lesión de LCA programados a cirugía con la subsecuente cuantificación objetiva de la maniobra de Lachmann y pivot-shift de ambas rodillas (sana y lesionada) utilizando artrómetros, esta medición se realizó previo a la cirugía y bajo efectos de la anestesia. Se realizó un análisis estadístico con normalización de los resultados obtenidos; se utilizó para tal efecto la sumatoria, promedio, desviación estándar y energía de los datos obtenidos con los acelerómetros.

Finalmente, se realizó una clasificación con los datos normalizados y las unidades resultantes para definir los grados de lesión tomando como base el KT-1000.

Todos los pacientes incluidos contaban con diagnóstico comprobado de lesión del LCA mediante maniobras clínicas y estudios de imagen. Además, accedieron a firmar el consentimiento informado para participar en estudio. Se decidió excluir pacientes que ya hayan sido intervenidos previamente por lesión de LCA, con enfermedades degenerativas crónicas de la rodilla afectada o que en su defecto fueran portadores de prótesis.

\section{Resultados}

Se incluyeron 36 pacientes con un rango de edad de 1260 años de edad. El promedio de edades es de 33.4 años. La población consta de $73.53 \%$ de hombres y $26.47 \%$ de mujeres. Todos ellos fueron evaluados utilizando la escala de Tegner-Lysholm y el cuestionario del IKDC, así como el KT-1000 y el pivot-shift meter con la finalidad de evaluar la laxitud y daño del LCA.

Para comparar los resultados con el pivot-shift meter se capturaron los resultados con el artrómetro KT-1000, debido a que es el «estándar de oro», encontrando un rango de 0 a $3 \mathrm{~mm}$ en $64 \%$ de los pacientes al restar al valor de la rodilla sana el valor de la rodilla afectada. Se encontró que $28 \%$ de los pacientes registraron valores de KT-1000 que oscilan entre los 4 y $6 \mathrm{~mm}$ de desplazamiento, lo que hizo evidente la lesión completa del LCA. En 8\% de los pacientes se encontraron valores de KT-1000 que superaron los $7 \mathrm{~mm}$ de desplazamiento. Con estos valores pudimos clasificar, cuantitativamente, la lesión de LCA de acuerdo al valor obtenido por KT-1000 en diferentes grados (Tabla 1).
Se analizaron los resultados de las gráficas obtenidas con el pivot-shift meter, en donde cada uno de los ejes fueron medidos por el dispositivo.

Se obtuvieron los resultados de la medición de 3 ejes de movimiento (X, Y y Z), de los cuales se obtuvieron integrales, para la medición más precisa de cada eje; lo anterior dio como resultado el eje Y como el más relevante para el estudio, ya que es en éste donde se pueden notar de forma más marcada las elevaciones previamente descritas como picos o espigas producidas por la luxación o subluxación femorotibial. Finalmente, se equipararon los resultados obtenidos en cada integral con los valores de sus respectivos KT-1000 para proponer una clasificación con grados de lesión.

Inicialmente, con los resultados del eje Y, se encontró que la integral de la velocidad de desplazamiento, mediante los acelerómetros (ACMs), fue acorde al grado de lesión dado por el artrómetro KT-1000 (estándar de oro). El 64\% de los pacientes clasificaron en lesión LCA grado 1 con KT-1000, registrándose, mediante ACMs, una diferencia en la integral de $\pm 750 \mathrm{~m} / \mathrm{s}$ entre pierna sana y lesionada $\sin$ reparar; recordemos que este grado representa las lesiones parciales. En los pacientes con grado 2 (28\%), la diferencia en la integral fue de hasta los $\pm 1,500 \mathrm{~m} / \mathrm{s}$ con ACMs; estos casos presentaron mayor lesión en el LCA, generalmente en sus dos haces, de tal manera que al reproducir el movimiento rotacional en la maniobra se detectó un mayor desplazamiento al no existir un ligamento íntegro. Finalmente, los pocos individuos que presentaron el grado 3 (8\%) alcanzaron una diferencia en integral de hasta $\pm 3,000 \mathrm{~m} / \mathrm{s}$, demostrando el mayor grado de lesión por la evidente rotura completa de los dos haces del LCA. Asociando las mediciones del KT-1000 y de pivot-shift meter sugerimos una nueva clasificación, a la que denominamos «del Parque» que permitirá cuantificar la maniobra de pivot-shift.

Tras un proceso de normalización de los datos obtenidos con el fin de eliminar variables por vibración o movilización del equipo, se obtuvieron diferentes resultados en cuanto a los diferentes ejes de movimiento en las maniobras realizadas. De acuerdo a los grados sugeridos en la clasificación con base en los resultados del KT-1000, se procedió a tomar en cuenta la sumatoria de los valores obtenidos en las maniobras ya normalizadas, de esta forma encontramos que en el grado 1 , el eje $\mathrm{X}$ se encuentra en un rango de 74-90 U en

\begin{tabular}{|c|c|c|c|c|}
\hline \multirow[b]{2}{*}{ Grado } & \multirow[b]{2}{*}{ KT-1000 (mm) } & \multicolumn{3}{|c|}{ Acelerómetros } \\
\hline & & Eje X & Eje Y & Eje Z \\
\hline 1 & $1-3$ & $75-90 *$ & $60-94^{*}$ & $60-74 *$ \\
\hline 2 & $4-6$ & $91-95^{*}$ & $95-100 *$ & $75-85^{*}$ \\
\hline 3 & $>7$ & $\geq 96^{*}$ & $\geq 100^{*}$ & $\geq 86^{*}$ \\
\hline
\end{tabular}


el grado 2 esta misma mostraba valores de 91-95 U y en el grado 3 los valores fluctuaban desde el 95 hasta las $100 \mathrm{U}$.

En cuanto al eje de las Y, los valores inician en el grado 1 con un rango de 63-94 U, grado 2 inicia de 95-100 U y finalmente en el grado 3 supera los 100 hasta las $124 \mathrm{U}$.

A continuación, en el eje de la Z, el grado 1 inicia en 60$74 \mathrm{U}$, el grado 2 de $75-85 \mathrm{U}$ y finalmente el grado 3 inicia de 86 hasta $105 \mathrm{U}$ (Tabla 2).

Posterior a ello, se elabora una clasificación «del Parque», en la que se muestran los diferentes grados establecidos, sus valores respectivos de KT-1000 y las unidades normalizadas (Tabla 3).

Tomando en cuenta las diferentes cifras que resultaron tras la sumatoria de las unidades obtenidas con el pivotshift meter, se toma en cuenta que mientras mayor sea el valor obtenido con el KT-1000 y por los acelerómetros, sin descartar la valoración clínica y la mecánica de la lesión, aumenta la probabilidad de la lesión parcial o completa del LCA. Tomando como base la recién propuesta clasificación, en los grados 1 es mayor la probabilidad de la lesión del haz anteromedial, encargado de la estabilidad anteroposterior. En el grado 2 se considera una lesión del haz anteromedial y posterolateral, ya que hay una mayor inestabilidad anteroposterior, así como un aumento en la inestabilidad rotacional. Finalmente, en el grado 3, encontramos una inestabilidad anteroposterior y rotacional considerable, por lo que se sugiere una reconstrucción de dos haces, tomando en cuenta la gran inestabilidad que encontramos en este caso.

\section{Discusión y conclusiones}

Con el presente estudio se consigue un análisis detallado del desarrollo de pivot-shift meter como un dispositivo que permite la valoración de lesiones parciales simples a rupturas completas del LCA, sin ser invasivo ni doloroso, por el contrario, la prueba clínica es rápida, práctica y con la ventaja de obtener resultados cuantitativos, así como cualitativos.

Se propone la clasificación de «del Parque», la cual toma como base la medición de la maniobra de Lachman para la estabilidad anteroposterior (KT-1000) y la de pivot-shift para la estabilidad rotacional mediante artrometría cuantificable. El propósito de la misma es establecer una diferencia cuantitativa entre los diferentes grados de lesión del LCA y clasificarlos de acuerdo a la severidad en grado 1, 2 y 3 . Además de esto, se propone en un trabajo futuro, relacionar los grados encontrados con un tipo de reconstrucción específica. Finalmente, presenta la ventaja de poder continuar con un control a mediano y largo plazo para valorar la estabilidad de la misma y su evolución tras la reconstrucción.

Dentro de las desventajas del estudio encontramos que es necesario unificar la técnica correcta para realizar la maniobra de pivot-shift, brindando instrucciones precisas para validar los resultados obtenidos mediante el uso de los artrómetros. En caso de no realizar la maniobra de la forma preestablecida, es posible encontrar falsos negativos o falsos positivos, por lo cual se debe estandarizar la manera de realizarla. Es necesario aumentar el número de muestra para una validez del estudio y una veracidad de los datos obtenidos.

En cuanto a modificaciones futuras, se espera lograr actualizar tanto hardware como software para la obtención de información más específica para un mejor diagnóstico y seguimiento, además de probables modificaciones futuras a la escala aquí propuesta para su actualización.

Referencias

1. Hughston JC, Andrews JR, Cross MJ, Moschi A. Classification of knee ligament instabilities. Part I. The medical compartment and cruciate ligaments. J Bone Joint Surg Am. 1976; 58(2): 159-72. Available in: https://doi.org/10.2106/00004623-197658020-00001

2. Aoyama JT, Lowe J, Capraro AC, Wells L. Clinical evaluation of ACL tear. The pediatric anterior cruciate ligament: evaluation and management strategies, 2017; 41-47. Available in: https://doi. org/10.1007/978-3-319-64771-5_6

3. Musahl V, Karlsson J. Anterior cruciate ligament tear. $N$ Engl J Med. 2019; 380(24): 2341-8. Available in: https://doi.org/10.1056/ NEJMcp1805931

4. Garín ZD, Reyes PE, Penagos PA. Lesión del ligamento cruzado anterior. Opciones actuales de tratamiento en el deportista. Orthotips. 2016; 12(2): 88-95.

5. Guía de Práctica Clínica, Manejo de las lesiones ligamentarias traumáticas en rodilla. México: Secretaría de Salud; 2009.

6. Berumen-Nafarrate E, Tonche-Ramos J, Carmona-González J, LealBerumen I, Vega-Nájera CA, Díaz-Arriaga JM, Espinoza-Sánchez MA, Aguirre-Madrid A. Interpretación de la maniobra de pivote mediante el uso de acelerómetros en pacientes que acuden a consulta ortopédica. Acta Ortop Mex. 2015; 29(3): 176-81.

7. Miller CD, Hogan MV, Miller MD. Bone-patellar tendon-bone ACL reconstruction. Master techniques in Orthpaedic Surgery, Sports Medicine Freddie Fu; 2010, Capitulo 26, 301-312.

8. Mae T, Shino K, Hiramatsu K, Tachibana Y, Nakagawa S, Yoshikawa $\mathrm{H}$. Anterior laxity of the knee assessed with gravity stress radiograph. Skeletal Radiol. 2018; 47(10): 1349-55. Available in: https://doi. org/10.1007/s00256-018-2941-5

9. Hooda A, Dhillon MS, Prabhakar S, Prakash M, John R, Kanwat H. MRI evaluation of anterolateral ligament tears in knee injury with anterior cruciate ligament rupture. Muscles, Ligaments and Tendons Journal. 2018; 8(1): 23-7. Available in: https://doi.org/10.11138/mltj/2018.8.1.023

10. Kircher J. Editorial commentary: the measurement of knee laxity: instrumented measurement and stress radiography for anterior cruciate ligament injuries. Arthroscopy - Journal of Arthroscopic and Related Surgery. 2019; 35(6): 1733-5. Available in: https://doi.org/10.1016/j. arthro.2019.03.015

11. Arcuri F, Abalo E, Barclay F. Uso de escores para evaluación de resultados en cirugía del ligamento cruzado anterior. Artroscopia. 2010; 17(3): 241-7.

12. Hambly K, Griva K, IKDC or KOOS? Which measures symptoms and disabilities most important to postoperative articular cartilage repair patients? Am J Sports Med. 2008; 36(9): 1695-704.

3. Huang W, Zhang Y, Yao Z, Ma L. Clinical examination of anterior cruciate ligament rupture: a systematic review and meta-analysis. Acta Orthop Traumatol Turc. 2016; 50(1): 22-31.

14. Bernard R, Bach Jr, Provencher MT, ACL surgery: how to get it right the first time and what to do if it fails. J Sports Sci Med. 2010; 9(3): 527. Available in: https://www.jssm.org/ Base de datos.

15. Kean BT, Burks RT. Diagnostic and surgical decision ACL tears. In: Bonnin M, Amendola A, Bellemans J, MacDonald S, Ménétrey J, editors. The knee joint: surgical techniques and strategies. [Internet]. Paris: Springer Paris; 2012. p. 151-61.

16. Kuroda R, Hoshino Y. Electromagnetic tracking of the pivot-shift. Curr Rev Musculoskelet Med. 2016; 9(2): 164-9. Available in: https:// doi.org/10.1007/s12178-016-9335-X 
17. Slichter ME, Wolterbeek N, Auw Yang KG, Zijl JAC, Piscaer TM. A novel test for assessment of anterolateral rotatory instability of the knee: the tibial internal rotation test (TIR test). J Exp Orthop. 2018; 5(1): 29. Available in: https://doi.org/10.1186/s40634-018-0141-9

18. Getgood A, Bryant D, Firth A. The stability study: a protocol for a multicenter randomized clinical trial comparing anterior cruciate ligament reconstruction with and without lateral extra-articular tenodesis in individuals who are at high risk of graft failure. $B M C$ Musculoskelet Disord. 2019; 20(1): 1-11. Available in: https://doi. org/10.1186/s12891-019-2589-x

19. Chung JH, Ryu KJ, Lee DH, Yoon KH, Park YW, Kim HJ, Kim $\mathrm{JH}$. An analysis of normative data on the knee rotatory profile and the usefulness of the Rotatometer, a new instrument for measuring tibiofemoral rotation: the reliability of the knee Rotatometer. Knee Surg Sports Traumatol Arthrosc. 2015; 23(9): 2727-33. Available in: https://doi.org/10.1007/s00167-014-3039-9

20. Ferretti A, Monaco E, Fabbri M, Maestri B, De Carli A. Prevalence and classification of injuries of anterolateral complex in acute anterior cruciate ligament tears. Arthroscopy 2017; 33(1): 147-54. Available in: https://doi.org/10.1016/j.arthro.2016.05.010

21. Tanaka T, Hoshino Y, Miyaji N, Ibaragi K, Nishida K, Nishizawa Y, et al. The diagnostic reliability of the quantitative pivot-shift evaluation using an electromagnetic measurement system for anterior cruciate ligament deficiency was superior to those of the accelerometer and iPad image analysis. Knee Surg Sports Traumatol Arthrosc. 2018; 26(9): 2835-40. Available in: https://doi.org/10.1007/s00167-0174734-0

22. Wheeless CR. Wheeless textbook in orthopaedics. 2016.

23. During NL, Lateral S, Sprain A, Weimar WH, State M. Note : This article will be published in a forthcoming issue of the Journal of Sport Rehabilitation. The article appears here in its accepted, peer-reviewed form, as it was provided by the submitting author. It has not been copyedited, proofed, or formatted by the publisher. Journal of Sport Rehabilitation. 2019.
24. Murgier J, Béranger JS, Boisrenoult P, Steltzlen C, Pujol N. Prospective comparative study of knee laxity with four different methods in anterior cruciate ligament tears. International Orthopaedics. 2018; 42(8): 1845-51. Available in: https://doi.org/10.1007/s00264-0183791-4

25. Svantesson E, Hamrin SE, Martensson J, Zaffagnini S, Kuroda R, Musahl V, et al. Static anteroposterior knee laxity tests are poorly correlated to quantitative pivot shift in the ACL-deficient knee: a prospective multicentre study. Journal of ISAKOS: Joint Disorders \& Orthopaedic Sports Medicine. 2018; 3(2): 83-8. Available in: https:// doi.org/10.1136/jisakos-2017-000196

26. Alqahtani Y, Murgier J, Beaufils P, Boisrenoult P, Steltzlen C, Pujol $\mathrm{N}$. Anterior tibial laxity using the GNRB ${ }^{\circledR}$ device in healthy knees. Knee. 2018; 25(1): 34-9. Available in: https://doi.org/10.1016/j. knee.2017.03.004

27. Rahnemai-Azar AA, Naendrup JH, Soni A, Olsen A, Zlotnicki J, Musahl V. Knee instability scores for ACL reconstruction. Curr Rev Musculoskelet Med. 2016; 9(2): 170-7. Available in: https://doi. org/10.1007/s12178-016-9339-6

28. Sundemo D, Blom A, Hoshino Y, Kuroda R, Lopomo NF, Zaffagnini S, Samuelsson K. Correlation between quantitative pivot shift and generalized joint laxity: a prospective multicenter study of ACL ruptures. Knee Surg Sports Traumatol Arthrosc. 2018; 26(8): 2362-70. Available in: https://doi.org/10.1007/s00167-017-4785-2

29. Marchand JB, Ruiz N, Coupry A, Bowen M, Robert H. Do graft diameter or patient age influence the results of ACL reconstruction? Knee Surg Sports Traumatol Arthrosc. 2016; 24(9): 2998-3004. Available in: https://doi.org/10.1007/s00167-015-3608-6

30. Fanelli GC, Orcutt DR, Edson CJ. The multiple-ligament injured knee: evaluation, treatment, and results. Arthroscopy. 2005; 21(4): 471-86.

Fuente de financiamiento: todos los gastos incurridos al realizarse el estudio fueron absorbidos por los autores del artículo. 